\title{
ASYMPTOTIC BEHAVIOR OF DIFFERENTIAL EQUATIONS DRIVEN BY PERIODIC AND RANDOM PROCESSES WITH SLOWLY DECAYING CORRELATIONS
}

\author{
RENAUD MARTY ${ }^{1}$
}

\begin{abstract}
We consider a differential equation with a random rapidly varying coefficient. The random coefficient is a Gaussian process with slowly decaying correlations and compete with a periodic component. In the asymptotic framework corresponding to the separation of scales present in the problem, we prove that the solution of the differential equation converges in distribution to the solution of a stochastic differential equation driven by a classical Brownian motion in some cases, by a fractional Brownian motion in other cases. The proofs of these results are based on the Lyons theory of rough paths. Finally we discuss applications in two physical situations.
\end{abstract}

Mathematics Subject Classification. 34F05, 60F05, 60G15.

Received May 6, 2004. Revised November 8, 2004 and February 9, 2005.

\section{INTRODUCTION}

Limit theorems are very useful for approximation problems in many situations, for instance in physics [7-9] or mathematics for finance [6]. We consider in this paper a random field $\left(Y^{\varepsilon}(t)\right)_{t \in[0,+\infty)}$ which is solution of the random differential equation:

$$
\left\{\begin{array}{l}
\frac{\mathrm{d} Y^{\varepsilon}}{\mathrm{d} t}(t)=\varepsilon m(t) F\left(Y^{\varepsilon}(t)\right) \text { for } t \in[0,+\infty), \\
Y^{\varepsilon}(t=0)=x_{0} \in \mathbb{R}^{d}
\end{array}\right.
$$

where $F$ is a smooth function, $m$ is a continuous, stationary and centered stochastic process and $\varepsilon>0$ is a small dimensionless parameter. For instance the vector $Y^{\varepsilon}$ can model the position of a particle driven by a random velocity field or a vector of prices in mathematics for finance. We want to study the asymptotic behavior of $Y^{\varepsilon}$ when $\varepsilon$ goes to 0 . Under boundedness conditions on $F$ and $m$, it is clear that $Y^{\varepsilon} \rightarrow x_{0}$ when $\varepsilon \rightarrow 0$. So our aim is to find the time scale which leads to a nontrivial effective evolution of the position of the particle or the vector of prices. In some cases it appears that the good scale is $t \rightarrow t / \varepsilon^{2}$. The rescaled quantity $X^{\varepsilon}:=Y^{\varepsilon}\left(\cdot / \varepsilon^{2}\right)$

\footnotetext{
Keywords and phrases. Limit theorems, stationary processes, rough paths.

1 Laboratoire de Statistique et Probabilités, Université Paul Sabatier, 118 route de Narbonne, 31062 Toulouse Cedex 4, France; marty@cict.fr 
satisfies the random differential equation:

$$
\left\{\begin{array}{l}
\frac{\mathrm{d} X^{\varepsilon}}{\mathrm{d} t}(t)=\frac{1}{\varepsilon} m\left(\frac{t}{\varepsilon^{2}}\right) F\left(X^{\varepsilon}(t)\right) \text { for } t \in[0,+\infty), \\
X^{\varepsilon}(t=0)=x_{0} \in \mathbb{R}^{d}
\end{array}\right.
$$

The limit of $X^{\varepsilon}$ when $\varepsilon \rightarrow 0$ is well known under prescribed sets of hypotheses, in particular on the mixing properties of $m$. For instance, we may assume that $m$ is an ergodic Markov process with generator $\mathcal{G}$ which satisfies the Fredholm alternative [23], or that $m$ is a $\phi$-mixing process with sufficiently decaying mixing function $\phi[12]$. Then, by classical approximation-diffusion theorem (see for instance [11,25,26] and the books by Kushner [12] and Ethier and Kurtz [5]) the solution $X^{\varepsilon}$ converges in distribution in the space of continuous functions to the diffusion process $X$ which is solution of the stochastic differential equation:

$$
\left\{\begin{array}{l}
\mathrm{d} X(t)=\sigma_{0} F(X(t)) \circ \mathrm{d} W(t) \text { for } t \in[0,+\infty), \\
X(t=0)=x_{0} \in \mathbb{R}^{d}
\end{array}\right.
$$

where $W$ is a classical Brownian motion $(\mathrm{cBm})$, o stands for the Stratonovich integration and $\sigma_{0}^{2}=$ $2 \int_{0}^{+\infty} \mathbb{E}[m(0) m(t)] \mathrm{d} t$ which is nonnegative by the Wiener-Kintchine theorem [22]. As a consequence, if $\sigma_{0}>0$, then we get a time scale which leads to a nontrivial evolution of $Y^{\varepsilon}$. A question that we can ask is: what does it happen if $\sigma_{0}=0$ ? The classical diffusion-approximation theorem still holds true and shows that $X^{\varepsilon}$ converges to 0 . So it seems that we should consider a longer scale to capture the effective behavior of $Y^{\varepsilon}$.

We would like to address another issue. The classical diffusion-approximation theorem requires the covariance function to be integrable. What does it happen if the covariance function of $m$ is not integrable? The influence of the random term is stronger, so it seems that we should consider a shorter scale to get the effective behavior of $Y^{\varepsilon}$.

In this article we address the two issues. We shall see that the suitable scale is not $t / \varepsilon^{2}$ anymore, but a longer scale in the case where $\sigma_{0}=0$ (the short-range case), and a smaller scale when the covariance function of $m$ is not integrable (the long-range case). We shall establish that the rescaled process $X^{\varepsilon}$ converges to the solution of a stochastic differential equation of the same type of (3), not driven by a classical Brownian motion, but driven by a fractional Brownian motion. The Hurst parameter of this fractional Brownian motion will turn out to depend on the decay rate of the covariance function. The Hurst parameter will be smaller than $1 / 2$ in the short-range case, and greater than $1 / 2$ in the long-range case. Note that numerous papers $[5,7,25,26]$ have examined the limit of differential equations driven by a scaled noise verifying some general conditions (that we can find for instance in Kushner's book [12]), and in all these papers the limit system is driven by a semi-martingale. So an original contribution of this paper is to show examples where the limit driving noise is not a semi-martingale.

Sometimes the dynamical system is driven by a forcing term resulting from the interplay between a random component and a periodic component [23]. For instance let us consider the system

$$
\left\{\begin{array}{l}
\frac{\mathrm{d} Y^{\varepsilon}}{\mathrm{d} t}(t)=\varepsilon m(t) \cos \left(\varepsilon^{b} t\right) F\left(Y^{\varepsilon}(t)\right) \text { for } t \in[0,+\infty), \\
Y^{\varepsilon}(t=0)=x_{0} \in \mathbb{R}^{d}
\end{array}\right.
$$

It is well-known $[5,12,25,26]$ that if $m$ is an ergodic Markov process with a generator which satisfies the Fredholm alternative, $b \in(0,2)$, then the rescaled quantity $X^{\varepsilon}=Y^{\varepsilon}\left(\cdot / \varepsilon^{2}\right)$ converges to the solution of (3) with $\sigma_{0}^{2}=\int_{0}^{+\infty} \mathbb{E}[m(0) m(t)] \mathrm{d} t$. We want to address the same issue as in the previous case, that is to say: what does it happen if the covariance function is not integrable or if the covariance function is integrable but suchthat $\int_{0}^{+\infty} \mathbb{E}[m(0) m(t)] \mathrm{d} t=0$ ? Do we get the same results as previously, that is to say the convergence to 
the solution of a stochastic differential equation driven by a fractional Brownian motion? The answer is no; we prove in this paper that $X^{\varepsilon}$ converges to the solution of a stochastic differential equation driven by a classical Brownian motion like in the classical case, but with a normalization which depends on the decay rate of the covariance function and on the frequency of the periodic component.

The proofs for the classical cases are based on the perturbed test function method $[5,12,25,26]$. This approach requires good mixing properties of the driving processes. These good mixing properties do not hold in our cases (in particular, the limit system is not always driven by a semi-martingale), so it seems that the perturbed test function method cannot be applied. We shall use the rough paths theory developed by Lyons (see $[15-17,19]$ for the theory and $[3,13,14]$ for some applications). Moreover we propose a new approach to the classical approximation-diffusion theorem using the rough paths theory.

In a previous work [20] we addressed the case where $m$ is a long-range process and we did not take into account a periodic component. In this paper we first extend this result to the short-range case, which requires deeper technical considerations, essentially due to the stronger roughness of the system. Second we consider the role of a periodic component on the evolution of the system. The interplay between randomness and periodicity leads to a diffusive asymptotic behavior, which is really different from the case without a periodic component. This is in dramatic contrast with the classical approximation diffusion situation where we get the same diffusive asymptotic behavior with or without periodic component.

The problem that we consider in this article is also triggered by some recent work [29] dealing with wave propagation in fractal random media. We expect that generalizations of our results will be obtained and applied to many situations: finance, wave propagation in random media, and other fields where dynamical systems are governed by differential equations with random forces. In this paper, we shall discuss applications in two physical situations.

The organization of the paper is as follows: Section 2 is a brief review of rough paths theory and stochastic differential equations driven by a fractional Brownian motion. In Section 3 we establish the main results which are proved in Section 4. In Section 5 we apply continuity theorem of Lyons to give a new proof of the classical approximation-diffusion theorem. Section 6 deals with generalizations of previous results in multi-dimensional cases. Finally we discuss in Section 7 some applications.

\section{SOME BACKGROUND}

\subsection{The fractional Brownian motion}

This section is a brief review of fractional Brownian motion properties. We refer the reader to [27] for a more exhaustive presentation.

Let $H \in(0,1)$. We call the fractional (one-dimensional) Brownian motion (fBm) with Hurst parameter $H$ the centered Gaussian process $\left(W_{H}(t)\right)_{t \in \mathbb{R}}$ with covariance function

$$
\mathbb{E}\left[W_{H}(s) W_{H}(t)\right]=\frac{1}{2}\left\{|t|^{2 H}+|s|^{2 H}-|t-s|^{2 H}\right\}
$$

Remark that if $H=1 / 2$, the process $W_{H}$ is the classical Brownian motion (cBm). However, if $H \neq 1 / 2, W_{H}$ is nor a semi-martingale, neither a Markov process. As a consequence, the construction for the fBm of a stochastic calculus turns out to be more tricky than for the $\mathrm{cBm}$. The aim of the following section is to give a short review of the method to construct solutions of stochastic differential equations driven by a fractional Brownian motion via rough paths theory introduced by Lyons [15-17,19] and used for instance in $[3,13]$. 


\subsection{Rough paths}

In this section we fix $p \geq 1$, and we consider only functions defined on $I:=[0,1]$. We say that a function $w: I \rightarrow \mathbb{R}^{n}$ is of $p$-finite variation if

$$
V_{p}(w):=\left(\sup _{D} \sum_{j=0}^{k-1}\left|w\left(t_{j+1}\right)-w\left(t_{j}\right)\right|^{p}\right)^{1 / p}<\infty
$$

where $\sup _{D}$ runs over all finite partitions $\left\{0=t_{0}<t_{1}<\cdots<t_{k}=1\right\}$ of $I$.

For any continuous function $w: I \rightarrow \mathbb{R}^{n}$ of finite variation, we construct the smooth rough path over $w$ :

$$
\begin{aligned}
& \widetilde{w}: \Delta \rightarrow \mathbb{R} \oplus \mathbb{R}^{n} \oplus\left(\mathbb{R}^{n}\right)^{\otimes 2} \oplus \cdots \oplus\left(\mathbb{R}^{n}\right)^{\otimes[p]} \\
&(s, t) \mapsto\left(1, \widetilde{w}_{1}(s, t), \widetilde{w}_{2}(s, t), \cdots, \widetilde{w}_{[p]}(s, t)\right),
\end{aligned}
$$

where $\Delta=\{0 \leq s \leq t \leq 1\}$ and $\widetilde{w}_{k}(s, t)$ is the $k$-th iterated integral of $w$ on $[s, t]$ :

$$
\widetilde{w}_{k}(s, t)=\int_{s<r_{1}<\cdots<r_{k}<t} \mathrm{~d} w\left(r_{1}\right) \otimes \cdots \otimes \mathrm{d} w\left(r_{k}\right) .
$$

The space of all smooth rough paths is endowed with the $p$-variation distance

$$
d_{p}(\widetilde{u}, \widetilde{v})=\sum_{j=1}^{[p]}\left(\sup _{D} \sum_{l}\left|\widetilde{u}_{j}\left(t_{l-1}, t_{l}\right)-\widetilde{v}_{j}\left(t_{l-1}, t_{l}\right)\right|^{p / j}\right)^{j / p}+\sup _{t \in[0,1]}|u(t)-v(t)|
$$

and is denoted by $\Omega_{p}^{\infty}$. The closure of this metric space is called the space of all geometric rough paths and is denoted by $\Omega_{p}$. One of the most important theorems of rough paths theory is the following one:

Theorem 1 (continuity theorem of Lyons). Let $G: \mathbb{R} \times \mathbb{R}^{d} \rightarrow \mathcal{L}\left(\mathbb{R}, \mathbb{R}^{d}\right)$, and $F: \mathbb{R} \times \mathbb{R}^{d} \rightarrow \mathcal{L}\left(\mathbb{R}^{n}, \mathbb{R}^{d}\right)$ be two smooth functions with all bounded derivatives up to the degree $[p]+1$. We consider $y$ the unique solution of the differential equation

$$
\mathrm{d} y(t)=G(t, y(t)) \mathrm{d} t+F(t, y(t)) \mathrm{d} w(t), \quad y(t=0)=y_{0},
$$

where $w$ is a finite variation function. Then the Itô map $\mathcal{I}: \widetilde{w} \mapsto \widetilde{y}$ is continuous with respect the p-variation distance from $\Omega_{p}^{\infty}\left(\mathbb{R}^{n}\right)$ to $\Omega_{p}^{\infty}\left(\mathbb{R}^{d}\right)$. Therefore there exists a unique extension of this map (that we still denote by $\mathcal{I})$ to the space $\Omega_{p}\left(\mathbb{R}^{n}\right)$.

This theorem is a consequence of the continuity theorem of Lyons (see $[3,13,15-17,19]$ ), where $G$ is assumed to be zero. Indeed, it suffices to replace the noise $w: t \mapsto w(t)$ by the noise $w^{*}: t \mapsto(t, w(t))$. Then, by a straithforward calculation using integrations by part, we show that the application $\widetilde{w} \mapsto \widetilde{w^{*}}$ is continuous in the space of geometric rough paths (see for instance [18] pp. 140-141).

\subsection{Stochastic differential equations driven by $\mathrm{fBm}$}

Let us consider the case $n=1$. We can check that for any continuous function $w: I \rightarrow \mathbb{R}$ of finite $p$-variation, there exists a geometric rough path $\widetilde{w}$ which has the explicit formulation:

$$
\widetilde{w}(s, t)=\left(1, w(t)-w(s), \frac{(w(t)-w(s))^{2}}{2}, \cdots, \frac{(w(t)-w(s))^{[p]}}{[p] !}\right) .
$$

We consider a fBm $W_{H}$ with Hurst parameter $H$. The sample paths are $H^{\prime}-$ Hölder continuous for all $H^{\prime}<H$, so they are of finite $p$-variation for all $p>1 / H$. Then there exists a geometric rough path for all sample paths 
of $W_{H}$, which we denote by $\widetilde{W_{H}}$. Following Theorem 1 we can define $\mathcal{I}\left(\widetilde{W_{H}}\right)_{1}+y_{0}$ as the strong solution of the stochastic differential equation driven by a $\mathrm{fBm}$ :

$$
\mathrm{d} y(t)=G(t, y(t)) \mathrm{d} t+F(t, y(t)) \circ \mathrm{d} W_{H}(t), y(t=0)=y_{0}
$$

Note that if $H=1 / 2$, the strong solution of (5) defined as above is the same as the strong solution defined by classical Stratonovich's calculus.

Now we consider the case where $n>1$. It is proved by Coutin and Qian in [3] that there exists a geometric rough path of order $p$ with $p>1 / H$ for all sample paths of a multi-dimensional $\mathrm{fBm}$ with Hurst index $H>1 / 4$. So we can construct in this case a strong solution of stochastic differential equations driven by such a fBm. It is also shown that this construction can be extended to more general Gaussian processes.

\section{MAin RESUlts}

\subsection{Convergence to the solution of a stochastic differential equation driven by a fractional Brownian motion}

In this section we consider the family of processes $\left(X^{\varepsilon}\right)_{\varepsilon}$ in $\mathbb{R}^{d}$ which is defined by:

$$
\left\{\begin{array}{l}
\frac{\mathrm{d} X^{\varepsilon}}{\mathrm{d} t}(t)=G\left(t, X^{\varepsilon}(t)\right)+\frac{1}{\varepsilon^{\alpha}} m\left(\frac{t}{\varepsilon^{2}}\right) F\left(t, X^{\varepsilon}(t)\right) \text { for } t \in[0,1] \\
X^{\varepsilon}(t=0)=x_{0} \in \mathbb{R}^{d}
\end{array}\right.
$$

where $G$ and $F$ are two smooth functions (strict conditions will be given below), $m$ is a continuous, stationary and centered stochastic process, and $\alpha \in(0,2)$. We denote by $r$ the covariance function of $m$, that is $r(t)=$ $\mathbb{E}[m(0) m(t)]$. We define the following assumptions:

Assumption $(\mathcal{H} 1): \alpha \in(0,1)$ and there exists $c_{0}>0$ such that $r(t) \sim c_{0} / t^{\alpha}$ when $t \rightarrow+\infty$.

Assumption $(\mathcal{H} 2): \alpha \in(1,2)$, there exists $c_{0}<0$ such that $r(t) \sim c_{0} / t^{\alpha}$ when $t \rightarrow+\infty$ and $\int_{0}^{+\infty} r(t) \mathrm{d} t=0$.

Before stating the main result of this section we give examples of processes which satisfy Assumption $(\mathcal{H} 1)$ or $(\mathcal{H} 2)$. Let $H \in(0,1)$. We call the fractional (one-dimensional) white noise with index $H$ the process $\left(m_{H}(t)\right)_{t \in \mathbb{R}}$ defined by

$$
m_{H}(t)=W_{H}(t+1)-W_{H}(t),
$$

where $W_{H}$ is a $\mathrm{fBm}$ with Hurst parameter $H$. This process is continuous, Gaussian, stationary, centered and his covariance function is given by

$$
\mathbb{E}\left[m_{H}(0) m_{H}(t)\right]=\frac{1}{2}\left\{|t+1|^{2 H}+|t-1|^{2 H}-2|t|^{2 H}\right\} .
$$

If we take $\alpha=2-2 H$, we can check by straightforward calculations that $m_{H}$ satisfies $(\mathcal{H} 1)$ if $H>1 / 2,(\mathcal{H} 2)$ if $H<1 / 2$.

We call the (stationary) fractional Orstein-Uhlenbeck process with index $H$ the process $\left(\nu_{H}(t)\right)_{t \in \mathbb{R}}$ defined by

$$
\nu_{H}(t)=W_{H}(t)-\mathrm{e}^{-t} \int_{-\infty}^{t} \mathrm{e}^{\theta} W_{H}(\theta) \mathrm{d} \theta
$$

where $W_{H}$ is a fBm with Hurst parameter $H$. As the white noise with index $H, \nu_{H}$ is continuous, Gaussian, stationary, centered and, taking $\alpha=2-2 H$, satisfies $(\mathcal{H} 1)$ if $H>1 / 2,(\mathcal{H} 2)$ if $H<1 / 2$. 
The main result of this section is the following.

Theorem 2. Assume that:

1) $m$ is Gaussian and $(\mathcal{H} 1)$ or $(\mathcal{H} 2)$ is satisfied.

2) $F$ and $G$ are two functions from $\mathbb{R} \times \mathbb{R}^{d}$ to $\mathbb{R}^{d}$ of class $\mathcal{C}^{[2 /(2-\alpha)]+1}$ with bounded derivatives.

Let $\left(X^{\varepsilon}\right)_{\varepsilon}$ be the family of processes which is solution of (6). When $\varepsilon$ goes to $0, X^{\varepsilon}$ converges in distribution in $\mathcal{C}\left([0,1], \mathbb{R}^{d}\right)$ to the process $X$ which is solution of:

$$
\left\{\begin{array}{l}
\mathrm{d} X(t)=G(t, X(t)) \mathrm{d} t+C_{0} F(t, X(t)) \circ \mathrm{d} W_{H}(t) \text { for } t \in[0,1], \\
X(t=0)=x_{0} \in \mathbb{R}^{d},
\end{array}\right.
$$

where $W_{H}$ is a fractional Brownian motion with Hurst index $H=(2-\alpha) / 2$ and $C_{0}^{2}=H^{-1}(2 H-1)^{-1} c_{0}$. In fact, the convergence holds in the space of geometric rough paths of order $p>2 /(2-\alpha)$.

Section 4.1 is devoted to the proof of this theorem.

\subsection{Convergence to the solution of a stochastic differential equation driven by a classical Brownian motion}

Now we consider the family of processes $\left(X^{\varepsilon}\right)_{\varepsilon}$ in $\mathbb{R}^{d}$ which is defined by:

$$
\left\{\begin{array}{l}
\frac{\mathrm{d} X^{\varepsilon}}{\mathrm{d} t}(t)=G\left(t, X^{\varepsilon}(t)\right)+\frac{1}{\varepsilon^{\gamma}} m\left(\frac{t}{\varepsilon^{2}}\right) \cos \left(\frac{t}{\varepsilon^{\beta}}\right) F\left(t, X^{\varepsilon}(t)\right) \text { for } t \in[0,1], \\
X^{\varepsilon}(t=0)=x_{0} \in \mathbb{R}^{d},
\end{array}\right.
$$

where $G$ and $F$ are two smooth functions (strict conditions will be given below), $m$ is a continuous, stationary and centered stochastic process, and $\gamma, \beta$ are two parameters. We denote by $r$ the covariance function of $m$. We define the following assumptions:

Assumption $(\mathcal{H} 3)$ : There exist $\alpha \in(0,1)$ and $c_{0}>0$ such that $r(t)=c_{0} / t^{\alpha}+f(t)$ where $f(t)=\mathcal{O}\left(t^{-\alpha-2}\right)$ when $t \rightarrow+\infty$.

Assumption $(\mathcal{H} 4)$ : There exist $\alpha \in(1,2)$ and $c_{0}<0$ such that $r(t)=c_{0} / t^{\alpha}+f(t)$ where $f(t)=\mathcal{O}\left(t^{-\alpha-2}\right)$ when $t \rightarrow+\infty$ and $\int_{0}^{+\infty} r(t) \mathrm{d} t=0$.

For instance the fractional white noise $m_{H}$ which is defined in the previous section satisfies Assumption $(\mathcal{H} 3)$ if $H>1 / 2$ and $(\mathcal{H} 4)$ if $H<1 / 2$. The main result of this section is the following.

Theorem 3. Assume that:

1) $m$ is Gaussian and $(\mathcal{H} 3)$ or $(\mathcal{H} 4)$ is satisfied.

2) $\beta \in(0,2)$ and $\gamma=1-(1-\alpha)(2-\beta) / 2$.

3) $F$ and $G$ are two smooth functions from $\mathbb{R} \times \mathbb{R}^{d}$ to $\mathbb{R}^{d}$ of class $\mathcal{C}^{[2 /(1-\alpha)]+1}$ with bounded derivatives if (H3) is satisfied. F and $G$ are two smooth functions from $\mathbb{R} \times \mathbb{R}^{d}$ to $\mathbb{R}^{d}$ of class $\mathcal{C}^{[2 /(2-\alpha)]+1}$ with bounded derivatives if $(\mathcal{H} 4)$ is satisfied.

Let $\left(X^{\varepsilon}\right)_{\varepsilon}$ be the family of processes which is solution of (8). When $\varepsilon$ goes to $0, X^{\varepsilon}$ converges in distribution in $\mathcal{C}\left([0,1], \mathbb{R}^{d}\right)$ to the process $X$ which is solution of

$$
\left\{\begin{array}{l}
\mathrm{d} X(t)=G(t, X(t)) \mathrm{d} t+C_{0} F(t, X(t)) \circ \mathrm{d} W(t) \text { for } t \in[0,1] \\
X(t=0)=x_{0} \in \mathbb{R}^{d}
\end{array}\right.
$$


where $W$ is a classical Brownian motion, $C_{0}^{2}=c_{0} \cos ((1-\alpha) \pi / 2) \Gamma(2-\alpha) /(1-\alpha)^{1}$. In fact, the convergence holds in the space of geometric rough paths of order $p>2 /(1-\alpha)$ for $\alpha \in(0,1)$, of order $p>2 /(2-\alpha)$ for $\alpha \in(1,2)$.

Sections 4.2 and 4.3 are devoted to the proof of this theorem.

\section{Proofs of the Main Results}

\subsection{Proof of Theorem 2}

The proof of Theorem 2 under $(\mathcal{H} 1)$ can be found in [20]. The proof of Theorem 2 under $(\mathcal{H} 2)$ follows the same lines, but it requires to study geometric rough paths of order $p$ with $p$ sufficiently large. This is essentially due to the strong roughness of the fractional Brownian motion of Hurst index $H<1 / 2$. Indeed, in the long-range case, $p=1$ is sufficient. So we take $p>1 / H$. We let

$$
w^{\varepsilon}(t):=\frac{1}{\varepsilon^{\alpha}} \int_{0}^{t} m\left(\frac{\theta}{\varepsilon^{2}}\right) \mathrm{d} \theta=\varepsilon^{2 H} \int_{0}^{t / \varepsilon^{2}} m(\theta) \mathrm{d} \theta,
$$

and we denote by $\widetilde{w^{\varepsilon}}$ the geometric rough path of $w^{\varepsilon}$ for $p$, that is

$$
\widetilde{w^{\varepsilon}}(s, t):=\left(\widetilde{w}_{1}^{\varepsilon}(s, t), \widetilde{w}_{2}^{\varepsilon}(s, t), \cdots, \widetilde{w}_{[p]}^{\varepsilon}(s, t)\right)
$$

where $\widetilde{w}_{1}^{\varepsilon}(s, t)=w^{\varepsilon}(t)-w^{\varepsilon}(s)$ and $\widetilde{w}_{n}^{\varepsilon}(s, t)=\left(\widetilde{w}_{1}^{\varepsilon}(s, t)\right)^{n} / n$ !. By Theorem 1 it is sufficient to show that $\widetilde{w}^{\varepsilon}$ converges in distribution when $\varepsilon$ goes to 0 to the geometric rough path of the $\mathrm{fBm} W_{H}$ for the metric $\mathrm{d}_{p}$ :

$$
\mathrm{d}_{p}(\widetilde{u}, \widetilde{v})=\sum_{j=1}^{[p]}\left(\sup _{D} \sum_{l}\left|\widetilde{u}_{j}\left(t_{l-1}, t_{l}\right)-\widetilde{v}_{j}\left(t_{l-1}, t_{l}\right)\right|^{p / j}\right)^{j / p}+\sup _{t \in[0,1]}|u(t)-v(t)| .
$$

The aim of the first lemma is to prove the convergence of the finite-dimensional distributions of $\widetilde{w}^{\varepsilon}$. Then we prove an estimate on the increments of $w^{\varepsilon}$ that we use to conclude the proof by the tightness of $\tilde{w}^{\varepsilon}$ for the distance $\mathrm{d}_{p}$.

Lemma 1. When $\varepsilon$ goes to 0 , the finite-dimensional distributions of $\left(\widetilde{w}^{\varepsilon}\right)_{\varepsilon>0}$ converge in law to the finitedimensional distributions of $\widetilde{C_{0}} \widetilde{W_{H}}$ where $C_{0}^{2}=H^{-1}(2 H-1)^{-1} c_{0}$ and $\widetilde{W_{H}}$ is the geometric rough path of the $f B m W_{H}$.

Proof. By the continuity of the map $x \mapsto\left(x, x^{2} / 2, \cdots, x^{[p]} /[p] !\right)$ and the fact that $m$ is Gaussian and centered, we only have to show that for all $s, t \in[0,1]$,

$$
\lim _{\varepsilon \rightarrow 0} \mathbb{E}\left[w^{\varepsilon}(s) w^{\varepsilon}(t)\right]=\frac{C_{0}^{2}}{2}\left\{|t|^{2 H}+|s|^{2 H}-|t-s|^{2 H}\right\} .
$$

It is a straightforward extension to the continuous case of the result 7.2.11 p. 337 of Samorodnitsky and Taqqu [27].

The following lemma is useful for the tightness of $\left(\widetilde{w}^{\varepsilon}\right)_{\varepsilon>0}$.

Lemma 2. There exists a positive constant $C$ such that

$$
\mathbb{E}\left[\left(w^{\varepsilon}(t)-w^{\varepsilon}(s)\right)^{2}\right] \leq C|t-s|^{2 H}
$$

for all $t, s$ in $[0,1]$ and $\varepsilon$ in $(0,1)$.

\footnotetext{
${ }^{1}$ In the statement of the theorem $\Gamma$ stands for the Gamma Euler function $\Gamma(x)=\int_{0}^{\infty} \mathrm{e}^{-t} t^{x-1} \mathrm{~d} t$.
} 
Proof. If we let $w(t):=\int_{0}^{t} m(\theta) \mathrm{d} \theta$ it is sufficient to show that

$$
\mathbb{E}\left[(w(t)-w(s))^{2}\right] \leq C|t-s|^{2 H} .
$$

Since $r(t) \sim c / t^{\alpha}$ when $t \rightarrow \infty$ and $\int_{0}^{\infty} r(t) \mathrm{d} t=0$, there exists a constant $C^{\prime}$ such that $\int_{0}^{A} r(t) \mathrm{d} t \sim C^{\prime} A^{1-\alpha}$ when $A \rightarrow \infty$, so there exists a positive constant $C^{\prime \prime}$ such that $\int_{0}^{A} r(t) \mathrm{d} t \leq C^{\prime \prime} A^{1-\alpha}$. Combining this inequality with the fact that

completes the proof.

$$
\mathbb{E}\left[(w(t)-w(s))^{2}\right]=2 \int_{0}^{t-s} \mathrm{~d} \theta \int_{0}^{\theta} r(\sigma) \mathrm{d} \sigma
$$

Now we can prove the tightness of $\left(\widetilde{w}^{\varepsilon}\right)_{\varepsilon>0}$.

Lemma 3. The family $\left(\widetilde{w}^{\varepsilon}\right)_{\varepsilon>0}$ is tight in the space of geometric rough paths for the metric $\mathrm{d}_{p}$ for all $p>1 / H$. Proof. Using Lemmas 2 and 13 establish the proof.

\subsection{Proof of Theorem 3 under $(\mathcal{H} 3)$}

In this proof we take $p>2 /(1-\alpha)$ and we denote

$$
w^{\varepsilon}(t)=\varepsilon^{1+(1-\alpha) b / 2} \int_{0}^{t / \varepsilon^{2}} \cos \left(\varepsilon^{b} \theta\right) m(\theta) \mathrm{d} \theta,
$$

where $b=2-\beta$. This following proof follows the same lines as the proof of Theorem 2 .

Lemma 4. For all $s, t \in[0,1]$, we have:

$$
\lim _{\varepsilon \rightarrow 0} \mathbb{E}\left[\left(w^{\varepsilon}(t)-w^{\varepsilon}(s)\right)^{2}\right]=C_{0}^{2}|t-s| .
$$

Proof. Let $s<t \in[0,1]$. We have

$$
\begin{aligned}
\mathbb{E}\left[\left(w^{\varepsilon}(t)-w^{\varepsilon}(s)\right)^{2}\right] & =\varepsilon^{2+(1-\alpha) b} \int_{s / \varepsilon^{2}}^{t / \varepsilon^{2}} \mathrm{~d} \theta \cos \left(\varepsilon^{b} \theta\right) \int_{s / \varepsilon^{2}}^{t / \varepsilon^{2}} \mathrm{~d} \sigma \cos \left(\varepsilon^{b} \sigma\right) r(\theta-\sigma) \\
& =\frac{1}{2}\left(I_{1}^{\varepsilon}(s, t)+I_{2}^{\varepsilon}(s, t)\right),
\end{aligned}
$$

where

$$
\begin{aligned}
& I_{1}^{\varepsilon}(s, t)=\varepsilon^{2+(1-\alpha) b} \int_{s / \varepsilon^{2}}^{t / \varepsilon^{2}} \mathrm{~d} \theta \int_{s / \varepsilon^{2}}^{t / \varepsilon^{2}} \mathrm{~d} \sigma \cos \left(\varepsilon^{b}(\theta-\sigma)\right) r(\theta-\sigma), \\
& I_{2}^{\varepsilon}(s, t)=\varepsilon^{2+(1-\alpha) b} \int_{s / \varepsilon^{2}}^{t / \varepsilon^{2}} \mathrm{~d} \theta \int_{s / \varepsilon^{2}}^{t / \varepsilon^{2}} \mathrm{~d} \sigma \cos \left(\varepsilon^{b}(\theta+\sigma)\right) r(\theta-\sigma) .
\end{aligned}
$$

We have

$$
I_{1}^{\varepsilon}(s, t)=2\left(I_{1,1}^{\varepsilon}(s, t)-I_{1,2}^{\varepsilon}(s, t)\right)
$$

where

$$
\begin{aligned}
& I_{1,1}^{\varepsilon}(s, t)=\varepsilon^{(1-\alpha) b}(t-s) \int_{0}^{(t-s) / \varepsilon^{2}} \mathrm{~d} \sigma \cos \left(\varepsilon^{b} \sigma\right) r(\sigma), \\
& I_{1,2}^{\varepsilon}(s, t)=\varepsilon^{2+(1-\alpha) b} \int_{0}^{(t-s) / \varepsilon^{2}} \mathrm{~d} \sigma \cos \left(\varepsilon^{b} \sigma\right) \sigma r(\sigma) .
\end{aligned}
$$


To prove this lemma, it is sufficient to show

$$
\begin{aligned}
& I_{1,1}^{\varepsilon}(s, t) \stackrel{\varepsilon \rightarrow 0}{\longrightarrow} C_{0}^{2}(t-s), \\
& I_{1,2}^{\varepsilon}(s, t) \stackrel{\varepsilon \rightarrow 0}{\longrightarrow} 0, \\
& I_{2}^{\varepsilon}(s, t) \stackrel{\varepsilon \rightarrow 0}{\longrightarrow} 0 .
\end{aligned}
$$

Let us begin by (10). We can write $I_{1,1}^{\varepsilon}=J_{1,1}^{\varepsilon}+K_{1,1}^{\varepsilon}$ where

$$
\begin{aligned}
& J_{1,1}^{\varepsilon}(s, t)=c_{0}(t-s) \int_{0}^{(t-s) / \varepsilon^{2-b}} \mathrm{~d} \sigma \cos (\sigma) \sigma^{-\alpha}, \\
& K_{1,1}^{\varepsilon}(s, t)=\varepsilon^{(1-\alpha) b}(t-s) \int_{0}^{(t-s) / \varepsilon^{2}} \mathrm{~d} \sigma \cos \left(\varepsilon^{b} \sigma\right) f(\sigma) .
\end{aligned}
$$

Since $b<2, J_{1,1}^{\varepsilon} \rightarrow c_{0}(t-s) \int_{0}^{\infty} \mathrm{d} \sigma \cos (\sigma) \sigma^{-\alpha}=c_{0}(t-s) \cos ((1-\alpha) \pi / 2) \Gamma(1-\alpha) .\left|K_{1,1}^{\varepsilon}(s, t)\right| \leq \varepsilon^{(1-\alpha) b}(t-$ s) $\int_{0}^{\infty} \mathrm{d} \sigma|f(\sigma)| \rightarrow 0$ because $\alpha<1$. So (10) is shown.

Let us prove (11). We can write $I_{1,2}^{\varepsilon}=J_{1,2}^{\varepsilon}+K_{1,2}^{\varepsilon}$ where

$$
\begin{aligned}
& J_{1,2}^{\varepsilon}(s, t)=c_{0} \varepsilon^{2-b} \int_{0}^{(t-s) / \varepsilon^{2-b}} \mathrm{~d} \sigma \cos (\sigma) \sigma^{1-\alpha}, \\
& K_{1,2}^{\varepsilon}(s, t)=\varepsilon^{2+(1-\alpha) b} \int_{0}^{(t-s) / \varepsilon^{2}} \mathrm{~d} \sigma \cos \left(\varepsilon^{b} \sigma\right) \sigma f(\sigma) .
\end{aligned}
$$

By an integration by part:

$$
\begin{aligned}
J_{1,2}^{\varepsilon}(s, t)= & c_{0} \varepsilon^{\alpha(2-b)} \sin \left((t-s) \varepsilon^{b-2}\right)(t-s)^{1-\alpha} \\
& -c_{0} \varepsilon^{2-b}(1-\alpha) \int_{0}^{(t-s) / \varepsilon^{2-b}} \mathrm{~d} \sigma \sin (\sigma) \sigma^{-\alpha},
\end{aligned}
$$

so we can easily check that $J_{1,2}^{\varepsilon} \rightarrow 0$ because $b<2$ and $0<\alpha<1$. Moreover $\left|K_{1,2}^{\varepsilon}(s, t)\right| \leq \varepsilon^{2+(1-\alpha) b} \int_{0}^{\infty}|\sigma f(\sigma)| \mathrm{d} \sigma$. The function $\sigma \mapsto \sigma f(\sigma)$ is integrable, then $K_{1,2}^{\varepsilon} \rightarrow 0$, so (11) is proved.

Let us complete the proof of the lemma by showing (12). We can write

$$
I_{2}^{\varepsilon}(s, t)=\varepsilon^{2+(1-\alpha) b} \iint_{A^{\varepsilon} \cup B^{\varepsilon} \cup C^{\varepsilon} \cup D^{\varepsilon}} \mathrm{d} x \mathrm{~d} y r(x) \cos \left(\varepsilon^{b}\left(y+2 s / \varepsilon^{2}\right)\right),
$$

where

$$
\begin{aligned}
& A^{\varepsilon}=\left\{(x, y) \in\left[0,(t-s) / \varepsilon^{2}\right] \times\left[0,(t-s) / \varepsilon^{2}\right]: x \leq y\right\}, \\
& B^{\varepsilon}=\left\{(x, y) \in\left[0,(t-s) / \varepsilon^{2}\right] \times\left[(t-s) / \varepsilon^{2}, 2(t-s) / \varepsilon^{2}\right]: y \leq-x+2(t-s) / \varepsilon^{2}\right\}, \\
& C^{\varepsilon}=\left\{(x, y) \in\left[-(t-s) / \varepsilon^{2}, 0\right] \times\left[(t-s) / \varepsilon^{2}, 2(t-s) / \varepsilon^{2}\right]: y \leq x+2(t-s) / \varepsilon^{2}\right\}, \\
& D^{\varepsilon}=\left\{(x, y) \in\left[-(t-s) / \varepsilon^{2}, 0\right] \times\left[0,(t-s) / \varepsilon^{2}\right]:-y \leq x\right\} .
\end{aligned}
$$

Using trigonometric formulas and the basic estimate $|\sin | \leq 1$, we can easily check that $J_{1,2}^{\varepsilon}(s, t) \rightarrow 0$. This shows (12) and concludes the proof of Lemma 4.

Lemma 5. There exists a constant $C>0$ such that for all $t$ and $s \in[0,1]$, and $\varepsilon \in(0,1)$

$$
\mathbb{E}\left[\left(w^{\varepsilon}(t)-w^{\varepsilon}(s)\right)^{2}\right] \leq C|t-s|^{1-\alpha}
$$


Proof. In this proof we adopt the same notations as previously. $C$ stands for a constant which may vary from line to line but remains independent of $s, t$, and $\varepsilon$. Following the proof of (10) we get that $\left|I_{1,1}^{\varepsilon}\right| \leq C \times|t-s| \leq$ $C \times|t-s|^{1-\alpha}$.

We remember that $I_{1,2}^{\varepsilon}=J_{1,2}^{\varepsilon}+K_{1,2}^{\varepsilon}$. We have

$$
J_{1,2}^{\varepsilon}(s, t)=c_{0} \varepsilon^{\alpha(2-b)} \sin \left((t-s) \varepsilon^{b-2}\right)(t-s)^{1-\alpha}-c_{0} \varepsilon^{2-b}(1-\alpha) \int_{0}^{(t-s) / \varepsilon^{2-b}} \mathrm{~d} \sigma \sin (\sigma) \sigma^{-\alpha} .
$$

The modulus of the first term of this sum is smaller than $C \times(t-s)^{1-\alpha}$. For the second term we introduce the function $g: A \mapsto \frac{1}{A} \int_{0}^{A} \sin (\sigma) \sigma^{-\alpha} \mathrm{d} \sigma . g$ is bounded because $\alpha \in(0,1)$, so

$$
\left|\varepsilon^{2-b}(1-\alpha) \int_{0}^{(t-s) / \varepsilon^{2-b}} \mathrm{~d} \sigma \sin (\sigma) \sigma^{-\alpha}\right| \leq C \times(t-s)^{1-\alpha},
$$

and $\left|J_{1,2}^{\varepsilon}\right| \leq C \times(t-s)^{1-\alpha}$. For $K_{1,2}^{\varepsilon}$ we introduce the function $h: A \mapsto \frac{1}{A} \int_{0}^{A}|\sigma f(\sigma)| \mathrm{d} \sigma . h$ is bounded because $\alpha \in(0,1)$, so $\left|K_{1,2}^{\varepsilon}\right| \leq C \times(t-s)^{1-\alpha}$ and $\left|I_{1,2}^{\varepsilon}\right| \leq C \times(t-s)^{1-\alpha}$. Finally, using the estimate on $r$ and $|\sin | \leq 1$, we easily check that $\left|I_{2}^{\varepsilon}\right| \leq C \times(t-s)^{1-\alpha}$. This concludes the proof.

Now we prove the tightness of $\left(\widetilde{w}^{\varepsilon}\right)_{\varepsilon>0}$.

Lemma 6. The family $\left(\widetilde{w}^{\varepsilon}\right)_{\varepsilon>0}$ is tight in the space of geometric rough paths for the metric $\mathrm{d}_{p}$ for all $p>2 /(1-\alpha)$.

Proof. Using Lemmas 5 and 13 establishes the proof.

\subsection{Proof of Theorem 3 under $(\mathcal{H} 4)$}

Substituting Lemma 7 for Lemma 4 and Lemma 8 for Lemma 5 in the previous proof, we get the proof of Theorem 3 under $(\mathcal{H} 4)$.

Lemma 7. For all $s, t \in[0,1]$, we have:

$$
\lim _{\varepsilon \rightarrow 0} \mathbb{E}\left[\left(w^{\varepsilon}(t)-w^{\varepsilon}(s)\right)^{2}\right]=C_{0}^{2}|t-s|
$$

Proof. Let $s<t \in[0,1]$. Proceeding and using the same notations as in the proof of Lemma 4 we have

$$
\mathbb{E}\left[\left(w^{\varepsilon}(t)-w^{\varepsilon}(s)\right)^{2}\right]=I_{1,1}^{\varepsilon}(s, t)-I_{1,2}^{\varepsilon}(s, t)+\frac{1}{2} I_{2}^{\varepsilon}(s, t) .
$$

Hence, it is sufficient to show

$$
\begin{aligned}
& I_{1,1}^{\varepsilon}(s, t) \stackrel{\varepsilon \rightarrow 0}{\longrightarrow} C_{0}^{2}(t-s), \\
& I_{1,2}^{\varepsilon}(s, t) \stackrel{\varepsilon \rightarrow 0}{\longrightarrow} 0, \\
& I_{2}^{\varepsilon}(s, t) \stackrel{\varepsilon \rightarrow 0}{\longrightarrow} 0 .
\end{aligned}
$$

Let us begin by (13). We can write

$$
I_{1,1}^{\varepsilon}(s, t)=(t-s)\left(J_{1}^{\varepsilon}(s, t)+J_{2}^{\varepsilon}(s, t)+J_{3}^{\varepsilon}(s, t)\right),
$$


where

$$
\begin{aligned}
& J_{1}^{\varepsilon}(s, t)=c_{0} \int_{0}^{(t-s) / \varepsilon^{2-b}} \mathrm{~d} \sigma(\cos (\sigma)-1) \sigma^{-\alpha}, \\
& J_{2}^{\varepsilon}(s, t)=\varepsilon^{(1-\alpha) b} \int_{0}^{(t-s) / \varepsilon^{2}} \mathrm{~d} \sigma\left(\cos \left(\varepsilon^{b} \sigma\right)-1\right) f(\sigma), \\
& J_{3}^{\varepsilon}(s, t)=\varepsilon^{(1-\alpha) b} \int_{0}^{(t-s) / \varepsilon^{2}} \mathrm{~d} \sigma r(\sigma) .
\end{aligned}
$$

In the following, $C$ stands for a constant which may vary from line to line but remains independent of $s, t$ and $\varepsilon . \quad J_{1}^{\varepsilon}(s, t) \rightarrow c_{0} \int_{0}^{\infty} \mathrm{d} \sigma(\cos (\sigma)-1) \sigma^{-\alpha}=c_{0} \sin ((2-\alpha) \pi / 2) \Gamma(1-\alpha)$ because $b<2$. We have $\left|J_{2}^{\varepsilon}(s, t)\right| \leq$ $\varepsilon^{(3-\alpha) b} \int_{0}^{\infty} \sigma^{2}|f(\sigma)| \rightarrow 0$ because $\sigma \rightarrow \sigma^{2} f(\sigma) \in L^{1}$ and $\alpha<2$. We have $J_{3}^{\varepsilon}(s, t)=\varepsilon^{(1-\alpha) b} \int_{0}^{(t-s) / \varepsilon^{2}} \mathrm{~d} \sigma r(\sigma)$. We have $r(\sigma) \sim_{\sigma \rightarrow \infty} C \times \sigma^{-\alpha}$ and $\int_{0}^{\infty} r=0$, therefore $\int_{0}^{A} r \sim_{A \rightarrow \infty} C \times A^{1-\alpha}$, so $J_{3}^{\varepsilon}(s, t) \sim_{\varepsilon \rightarrow 0} C \times \varepsilon^{(b-2)(1-\alpha)}$. This proves (13).

Let us show (14). We can write

$$
I_{1,2}^{\varepsilon}(s, t)=J_{1,2}^{\varepsilon}(s, t)+K_{1,2}^{\varepsilon}(s, t)
$$

where

$$
\begin{aligned}
& J_{1,2}^{\varepsilon}(s, t)=\varepsilon^{2-b} \int_{0}^{(t-s) / \varepsilon^{2-b}} \mathrm{~d} \sigma \cos (\sigma) \sigma^{1-\alpha}, \\
& K_{1,2}^{\varepsilon}(s, t)=\varepsilon^{2+(1-\alpha) b} \int_{0}^{(t-s) / \varepsilon^{2}} \mathrm{~d} \sigma \cos \left(\varepsilon^{b} \sigma\right) \sigma f(\sigma) .
\end{aligned}
$$

The definite integral $\int_{0}^{\infty} \mathrm{d} \sigma \cos (\sigma) \sigma^{1-\alpha}$ exists and $b<2$, so $J_{1,2}^{\varepsilon}(s, t) \rightarrow 0$. Moreover $\left|K_{1,2}^{\varepsilon}(s, t)\right| \leq$ $\varepsilon^{2+(1-\alpha) b} \int_{0}^{(t-s) / \varepsilon^{2}} \mathrm{~d} \sigma|\sigma f(\sigma)| \cdot \int_{0}^{\infty}|\sigma f(\sigma)| \mathrm{d} \sigma<\infty$ and $2>b(\alpha-1)$, therefore $J_{1,2}^{\varepsilon}(s, t) \rightarrow 0$. This proves $(14)$.

The proof of (15) is similar to the proof of $(12)$.

Lemma 8. There exists a constant $C>0$ such that for all $t$ and $s \in[0,1]$, and $\varepsilon \in(0,1)$

$$
\mathbb{E}\left[\left(w^{\varepsilon}(t)-w^{\varepsilon}(s)\right)^{2}\right] \leq C|t-s|^{2-\alpha}
$$

Proof. The proof follows the same lines as the proof of Lemma 5 and essentially uses the calculations of the proof of Lemma 7 .

\section{Approximation-Diffusion VIA ROUGH PATHS}

In this section we use our previous arguments, particularly the theorem of continuity of Lyons to give a new proof of a classical approximation-diffusion theorem.

Theorem 4. Consider $p>2$. Let $F$ and $G$ two functions from $\mathbb{R} \times \mathbb{R}^{d}$ to $\mathbb{R}^{d}$ of class $\mathcal{C}^{3}$ with bounded derivatives and $m$ be a real-valued Gaussian, centered, continuous and stationary random process with covariance function $r(t)=\mathbb{E}[m(0) m(t)]$. We assume that $r$ is integrable and we let $C_{0}^{2}=\int_{0}^{\infty} r(t) \mathrm{d} t$. Then, when $\varepsilon$ goes to 0 ,

(i) the geometric rough path of order $p$ of the solution $X_{1}^{\varepsilon}$ of the problem

$$
\left\{\begin{array}{l}
\frac{\mathrm{d} X_{1}^{\varepsilon}}{\mathrm{d} t}(t)=G\left(t, X_{1}^{\varepsilon}(t)\right)+\frac{1}{\varepsilon} m\left(\frac{t}{\varepsilon^{2}}\right) F\left(t, X_{1}^{\varepsilon}(t)\right) \text { for } t \in[0,1], \\
X_{1}^{\varepsilon}(t=0)=x_{0} \in \mathbb{R}^{d}
\end{array}\right.
$$


converges in distribution in the space $\Omega_{p}$ to the geometric rough path of order $p$ of the solution $X_{1}$ of the stochastic differential equation

$$
\left\{\begin{array}{l}
\mathrm{d} X_{1}(t)=G\left(t, X_{1}(t)\right) \mathrm{d} t+\sqrt{2} C_{0} F\left(t, X_{1}(t)\right) \circ \mathrm{d} W(t) \text { for } t \in[0,1], \\
X_{1}(t=0)=x_{0} \in \mathbb{R}^{d},
\end{array}\right.
$$

where $W$ is a one-dimensional classical Brownian motion,

(ii) if $\beta \in] 0,2\left[\right.$, the geometric rough path of order $p$ of the solution $X_{2}^{\varepsilon}$ of the problem

$$
\left\{\begin{array}{l}
\frac{\mathrm{d} X_{2}^{\varepsilon}}{\mathrm{d} t}(t)=G\left(t, X_{2}^{\varepsilon}(t)\right)+\frac{1}{\varepsilon} m\left(\frac{t}{\varepsilon^{2}}\right) \cos \left(\frac{t}{\varepsilon^{\beta}}\right) F\left(t, X_{2}^{\varepsilon}(t)\right) \text { for } t \in[0,1], \\
X_{2}^{\varepsilon}(t=0)=x_{0} \in \mathbb{R}^{d},
\end{array}\right.
$$

converge in distribution in the space $\Omega_{p}$ to the geometric rough path of order $p$ of the solution $X_{2}$ of the stochastic differential equation

$$
\left\{\begin{array}{l}
\mathrm{d} X_{2}(t)=G\left(t, X_{2}(t)\right) \mathrm{d} t+C_{0} F\left(t, X_{2}(t)\right) \circ \mathrm{d} W(t) \text { for } t \in[0,1], \\
X_{2}(t=0)=x_{0} \in \mathbb{R}^{d} .
\end{array}\right.
$$

The $p$-variation topology is stronger than the uniform topology, so that this result covers the standard formulation of the approximation-diffusion theorem.

We let

$$
w_{1}^{\varepsilon}(t)=\varepsilon \int_{0}^{t / \varepsilon^{2}} m(\theta) \mathrm{d} \theta \text { and } w_{2}^{\varepsilon}(t)=\varepsilon \int_{0}^{t / \varepsilon^{2}} \cos \left(\varepsilon^{2-\beta} \theta\right) m(\theta) \mathrm{d} \theta .
$$

First we establish two preliminary lemmas.

Lemma 9. For all $s$ and $t$ in $[0,1]$, we have:

$$
\lim _{\varepsilon \rightarrow 0} \mathbb{E}\left[\left(w_{1}^{\varepsilon}(t)-w_{1}^{\varepsilon}(s)\right)^{2}\right]=2 C_{0}^{2}|t-s| \text { and } \lim _{\varepsilon \rightarrow 0} \mathbb{E}\left[\left(w_{2}^{\varepsilon}(t)-w_{2}^{\varepsilon}(s)\right)^{2}\right]=C_{0}^{2}|t-s| .
$$

Proof. The proof consists in some algebra using trigonometric formulas, the dominated convergence theorem and the fact that $r$ is integrable.

Lemma 10. For all $s$ and $t$ in $[0,1]$, there exists a constant $C>0$ such that for all $\varepsilon \in(0,1]$ :

$$
\mathbb{E}\left[\left(w_{1}^{\varepsilon}(t)-w_{1}^{\varepsilon}(s)\right)^{2}\right] \leq C|t-s| \text { and } \mathbb{E}\left[\left(w_{2}^{\varepsilon}(t)-w_{2}^{\varepsilon}(s)\right)^{2}\right] \leq C|t-s| .
$$

Proof. The proof essentially uses the calculations and the arguments of the previous proof.

Now we can prove Theorem 4.

Proof of Theorem 4. Substituting Lemma 9 for Lemma 4 and Lemma 10 for Lemma 5 in the proof of Theorem 3 under $(\mathcal{H} 3)$, we get the proof of Theorem 4.

\section{About the multi-Dimensional CASE}

\subsection{Extension of Theorem 2}

We consider the following problem:

$$
\left\{\begin{array}{l}
\frac{\mathrm{d} X^{\varepsilon}}{\mathrm{d} t}(t)=G\left(t, X^{\varepsilon}(t)\right)+\frac{1}{\varepsilon} \sum_{j=1}^{k} m_{j}\left(\frac{t}{\varepsilon^{2}}\right) F_{j}\left(t, X^{\varepsilon}(t)\right) \text { for } t \in[0,1], \\
X^{\varepsilon}(t=0)=x_{0} \in \mathbb{R}^{d}
\end{array}\right.
$$


where $m_{1}, \ldots, m_{k}$ are centered stochastic processes, $\varepsilon$ is a small parameter, and $G, F_{1}, \ldots, F_{k}$ are smooth functions. This type of equation has some applications, for instance in the study of light propagation in a birefringent optical fiber [10]. It is well-known $[25,26]$ that if the multi-dimensional process $\left(m_{1}, \ldots, m_{k}\right)$ is continuous, centered, Markovian, ergodic with a generator which satisfies the Fredholm alternative, and satisfying good correlations conditions (for instance the $m_{j}$ 's are independent), then the solution $X^{\varepsilon}$ converges in distribution to a limit $X$ which is solution of the stochastic differential equation

$$
\left\{\begin{array}{l}
\mathrm{d} X(t)=G(t, X(t)) \mathrm{d} t+\sum_{j=1}^{k} C_{j} F_{j}(t, X(t)) \circ \mathrm{d} W_{j}(t) \text { for } t \in[0,1] \\
X(t=0)=x_{0} \in \mathbb{R}^{d}
\end{array}\right.
$$

where the $W_{j}$ 's are independent standard Brownian motions and $C_{j}^{2}=2 \int_{0}^{+\infty} \mathbb{E}\left[m_{j}(0) m_{j}(t)\right] \mathrm{d} t$. The natural question that we can ask is: can we extend this result to the case where the $m_{j}$ 's satisfy the assumptions of Theorem 2, that is to say, can we establish a multi-dimensional version of Theorem 2? The following theorem establishes that the answer to this question is positive at least in the case where the $m_{j}$ 's are long-range processes. The case where the $m_{j}$ 's are short-range processes is more complicated and will be discussed at the end of this section.

Theorem 5. Assume that:

1) the $m_{j}$ 's are centered, stationary, Gaussian, independent processes;

2) there exist positive constants $c_{1}, c_{2}, \ldots, c_{k}$ and $1>\alpha_{1} \geq \alpha_{2} \geq \ldots \geq \alpha_{k}>0$ such that $\mathbb{E}\left[m_{j}(0) m_{j}(t)\right] \sim$ $c_{j} / t^{\alpha_{j}}$

3) the $F_{j}$ 's and $G$ are functions from $\mathbb{R} \times \mathbb{R}^{d}$ to $\mathbb{R}^{d}$ of class $\mathcal{C}^{\left[2 /\left(2-\alpha_{1}\right)\right]+1}$ with bounded derivatives. Then, when $\varepsilon$ goes to 0 , the solution $X^{\varepsilon}$ of the equation

$$
\left\{\begin{array}{l}
\frac{\mathrm{d} X^{\varepsilon}}{\mathrm{d} t}(t)=G\left(t, X^{\varepsilon}(t)\right)+\sum_{j=1}^{k} \frac{1}{\varepsilon^{\alpha_{j}}} m_{j}\left(\frac{t}{\varepsilon^{2}}\right) F_{j}\left(t, X^{\varepsilon}(t)\right) \text { for } t \in[0,1] \\
X^{\varepsilon}(t=0)=x_{0} \in \mathbb{R}^{d}
\end{array}\right.
$$

converge in distribution in $\mathcal{C}\left([0,1], \mathbb{R}^{d}\right)$ to the process $X$ which is solution of:

$$
\left\{\begin{array}{l}
\mathrm{d} X(t)=G(t, X(t)) \mathrm{d} t+\sum_{j=1}^{k} C_{j} F_{j}(t, X(t)) \circ \mathrm{d} W_{H_{j}}(t) \text { for } t \in[0,1] \\
X(t=0)=x_{0} \in \mathbb{R}^{d},
\end{array}\right.
$$

where the $W_{H_{j}}$ 's are independent fractional Brownian motions with Hurst indices $H_{j}=\left(2-\alpha_{j}\right) / 2$ and $C_{j}^{2}=H_{j}^{-1}\left(2 H_{j}-1\right)^{-1} c_{j}$. In fact, the convergence holds in the space of geometric rough paths of order $p>2 /\left(2-\alpha_{1}\right)$.

Proof. We fix $p \in\left(1 / H_{1}, 2\right)$. Following the continuity theorem of T. Lyons, it is sufficient to show that the geometric functional of order $1 \widetilde{w^{\varepsilon}}$ of $w^{\varepsilon}$,

$$
w^{\varepsilon}(t)=\left(\int_{0}^{t} \frac{1}{\varepsilon^{\alpha_{1}}} m_{1}\left(\frac{\theta}{\varepsilon^{2}}\right) \mathrm{d} \theta, \ldots, \int_{0}^{t} \frac{1}{\varepsilon^{\alpha_{k}}} m_{k}\left(\frac{\theta}{\varepsilon^{2}}\right) \mathrm{d} \theta\right)
$$


converges to the geometric functional of order 1 of the multi-dimensional fractional Brownian motion $\left(W_{H_{1}}, \ldots, W_{H_{k}}\right)$ for the $p$-variation distance $d_{p}$. The functional $\widetilde{w^{\varepsilon}}$ can write as

$$
\widetilde{w}^{\varepsilon}(s, t)=\left(\int_{s}^{t} \frac{1}{\varepsilon^{\alpha_{1}}} m_{1}\left(\frac{\theta}{\varepsilon^{2}}\right) \mathrm{d} \theta, \ldots, \int_{s}^{t} \frac{1}{\varepsilon^{\alpha_{k}}} m_{k}\left(\frac{\theta}{\varepsilon^{2}}\right) \mathrm{d} \theta\right),
$$

where all components are independent. So, we have just to prove for all $j=1, \ldots, k$ the convergence of $\int_{s}^{t} \frac{1}{\varepsilon^{\alpha_{j}}} m_{j}\left(\frac{\theta}{\varepsilon^{2}}\right) \mathrm{d} \theta$. This is already proved in the proof of Theorem 2 under $(\mathcal{H} 1)$.

We can imagine multiple extensions of Theorem 5 . One of the most natural extensions is a multi-dimensional version of Theorem 2 under $(\mathcal{H} 2)$. The problem is that the roughness of the possible limit implies that we have to prove the convergence of the iterated integrals to the iterated integrals of $\mathrm{fBm}$, which cannot be written as continuous real-valued functions of Gaussian variables as in proof of Theorem 2 under $(\mathcal{H} 2)$. This is an interesting issue that deserves further analysis.

\subsection{Extension of Theorem 3}

We consider the complex-valued vector $X^{\varepsilon}$ which is solution of the following problem:

$$
\left\{\begin{array}{l}
\frac{\mathrm{d} X^{\varepsilon}}{\mathrm{d} t}(t)=G\left(t, X^{\varepsilon}(t)\right)+\frac{1}{\varepsilon^{\gamma}} m\left(\frac{t}{\varepsilon^{2}}\right) \exp \left(i \frac{t}{\varepsilon^{\beta}}\right) F\left(t, X^{\varepsilon}(t)\right) \text { for } t \in[0,1], \\
X^{\varepsilon}(t=0)=x_{0} \in \mathbb{C}^{d}
\end{array}\right.
$$

This type of equations has a lot of applications in various physical domains, particularly in the study of wave propagation in random media [7-9,23]. It is well-known $[25,26]$ that if the process $m$ is continuous, centered, Markovian, ergodic with a generator which satisfies the Fredholm alternative, $\gamma=1$ and $\beta \in(0,2)$, then the solution $X^{\varepsilon}$ converges in distribution to a limit $X$ which is solution of the stochastic differential equation

$$
\left\{\begin{array}{l}
\mathrm{d} X(t)=G(t, X(t)) \mathrm{d} t+C_{0} F(t, X(t)) \circ\left(\mathrm{d} W_{1}(t)+i \mathrm{~d} W_{2}(t)\right) \text { for } t \in[0,1], \\
X(t=0)=x_{0} \in \mathbb{C}^{d},
\end{array}\right.
$$

where $W_{1}$ and $W_{2}$ are two independent standard Brownian motions and $C_{0}=\int_{0}^{\infty} \mathbb{E}[m(0) m(t)] \mathrm{d} t$ is a nonnegative constant. We can ask the question: in the same way as for Theorem 3, can we prove such a result under Assumption $(\mathcal{H} 3)$ or $(\mathcal{H} 4)$ ? This can be expected but we prove in this section a weaker result which is sufficient for some applications (see Sect. 7). We denote

$$
\begin{aligned}
w_{c}^{\varepsilon}(t) & =\varepsilon^{1+(1-\alpha)(2-\beta) / 2} \int_{0}^{t / \varepsilon^{2}} \cos \left(\varepsilon^{2-\beta} \theta\right) m(\theta) \mathrm{d} \theta, \\
w_{s}^{\varepsilon}(t) & =\varepsilon^{1+(1-\alpha)(2-\beta) / 2} \int_{0}^{t / \varepsilon^{2}} \sin \left(\varepsilon^{2-\beta} \theta\right) m(\theta) \mathrm{d} \theta .
\end{aligned}
$$

Theorem 6. We assume $m$ to be Gaussian, $(\mathcal{H} 3)$ or $(\mathcal{H} 4)$ to be satisfied with $\beta \in(0,2)$ and $\gamma=1-$ $(1-\alpha)(2-\beta) / 2$. Then, the sequence of stochastic fields $\left(w_{c}^{\varepsilon}(\cdot), w_{s}^{\varepsilon}(\cdot)\right)$ converges in distribution in $\mathcal{C}\left([0,1], \mathbb{R}^{2}\right)$ to $C_{0} \mathbf{W}$ where $\mathbf{W}$ is a two-dimensional standard Brownian motion, $C_{0}^{2}=c_{0} \cos ((1-\alpha) \pi / 2) \Gamma(2-\alpha) /(1-\alpha)$.

Proof. The proof of this theorem is an extension of the proofs of Lemmas 4, 5, 7 and 8 . In fact, it remains to prove that for all $s$ and $t, \mathbb{E}\left[w_{c}^{\varepsilon}(t) w_{s}^{\varepsilon}(s)\right]$ converge to 0 when $\varepsilon$ goes to 0 , to ensure the independence of the two coordinates of the limit process. We begin by the case where $t=s$. By a trigonometric formula we can write

$$
\mathbb{E}\left[w_{c}^{\varepsilon}(t) w_{s}^{\varepsilon}(t)\right]=\frac{1}{2}\left(L_{1}^{\varepsilon}(t)+L_{2}^{\varepsilon}(t)\right),
$$


where

$$
\begin{aligned}
& L_{1}^{\varepsilon}(t)=\varepsilon^{2+(1-\alpha)(2-\beta)} \int_{0}^{t / \varepsilon^{2}} \mathrm{~d} \theta \int_{0}^{t / \varepsilon^{2}} \mathrm{~d} \sigma \sin \left(\varepsilon^{2-\beta}(\sigma-\theta)\right) r(\sigma-\theta), \\
& L_{2}^{\varepsilon}(t)=\varepsilon^{2+(1-\alpha)(2-\beta)} \int_{0}^{t / \varepsilon^{2}} \mathrm{~d} \theta \int_{0}^{t / \varepsilon^{2}} \mathrm{~d} \sigma \sin \left(\varepsilon^{2-\beta}(\sigma+\theta)\right) r(\sigma-\theta) .
\end{aligned}
$$

To prove that $L_{2}^{\varepsilon}(t)$ converge to 0 , we proceed as for $I_{2}^{\varepsilon}(s, t)$ in the previous proofs. For $L_{1}^{\varepsilon}(t)$, we perform the change of variable $(\theta, \sigma) \rightarrow\left(t / \varepsilon^{2}-\theta, t / \varepsilon^{2}-\sigma\right)$ and using the fact that $\sin (-x)=-\sin (x)$ and $r(x)=r(-x)$, we get that $L_{1}^{\varepsilon}(t)=0$.

Now we assume that $s \neq t$, for instance $s<t$. From the result obtained in the case $t=s$, it suffices to show that

$$
L_{3}^{\varepsilon}(s, t):=\mathbb{E}\left[\left(w_{c}^{\varepsilon}(t)-w_{c}^{\varepsilon}(s)\right) w_{s}^{\varepsilon}(s)\right] \stackrel{\varepsilon \rightarrow 0}{\longrightarrow} 0 .
$$

Let us prove $(22)$ under $(\mathcal{H} 4)$. Note that:

$$
L_{3}^{\varepsilon}(s, t)=\varepsilon^{2+(1-\alpha)(2-\beta)} \int_{0}^{s / \varepsilon^{2}} \mathrm{~d} \sigma \sin \left(\varepsilon^{b} \sigma\right) \int_{s / \varepsilon^{2}}^{t / \varepsilon^{2}} \mathrm{~d} \theta \cos \left(\varepsilon^{b} \theta\right) r(\theta-\sigma) .
$$

Then, we get:

$$
\begin{aligned}
L_{3}^{\varepsilon}(s, t) & \leq \varepsilon^{2+(1-\alpha)(2-\beta)} \int_{0}^{s / \varepsilon^{2}} \mathrm{~d} \sigma \int_{s / \varepsilon^{2}}^{t / \varepsilon^{2}} \mathrm{~d} \theta|r(\theta-\sigma)| \\
& \leq \varepsilon^{2+(1-\alpha)(2-\beta)} C \int_{0}^{s / \varepsilon^{2}} \mathrm{~d} \sigma \int_{s / \varepsilon^{2}}^{t / \varepsilon^{2}} \mathrm{~d} \theta(\theta-\sigma)^{-\alpha} \\
& =\frac{\varepsilon^{(\alpha-1) \beta}}{(\alpha-1)(2-\alpha)} C\left((t-s)^{2-\alpha}-t^{2-\alpha}+s^{2-\alpha}\right) \stackrel{\varepsilon \rightarrow 0}{\longrightarrow} 0,
\end{aligned}
$$

because we assume $(\mathcal{H} 4)$.

Now we prove $(22)$ under $(\mathcal{H} 3)$. By a trigonometric formula we can write

$$
L_{3}^{\varepsilon}(s, t)=\frac{1}{2}\left(L_{3,1}^{\varepsilon}(s, t)+L_{3,2}^{\varepsilon}(s, t)\right)
$$

where

$$
\begin{aligned}
& L_{3,1}^{\varepsilon}(s, t)=\varepsilon^{2+(1-\alpha)(2-\beta)} \int_{s / \varepsilon^{2}}^{t / \varepsilon^{2}} \mathrm{~d} \theta \int_{0}^{s / \varepsilon^{2}} \mathrm{~d} \sigma \sin \left(\varepsilon^{b}(\theta-\sigma)\right) r(\theta-\sigma), \\
& L_{3,2}^{\varepsilon}(s, t)=\varepsilon^{2+(1-\alpha)(2-\beta)} \int_{s / \varepsilon^{2}}^{t / \varepsilon^{2}} \mathrm{~d} \theta \int_{0}^{s / \varepsilon^{2}} \mathrm{~d} \sigma \sin \left(\varepsilon^{b}(\theta+\sigma)\right) r(\theta-\sigma) .
\end{aligned}
$$

$L_{3,2}^{\varepsilon}(s, t)$ can be treated exactly as $I_{2}^{\varepsilon}(s, t)$ in the proof of Lemma 4 . It remains to deal with $L_{3,1}^{\varepsilon}(s, t)$. By a change of variable we get:

$$
\begin{aligned}
L_{3,1}^{\varepsilon}(s, t) & =\varepsilon^{2+(1-\alpha)(2-\beta)} \int_{s / \varepsilon^{2}}^{t / \varepsilon^{2}} \mathrm{~d} \theta \int_{\theta-s / \varepsilon^{2}}^{\theta} \mathrm{d} \sigma \sin \left(\varepsilon^{b} \sigma\right) r(\sigma) \\
& =M^{\varepsilon}(t)-M^{\varepsilon}(s)-M^{\varepsilon}(t-s),
\end{aligned}
$$


where

$$
M^{\varepsilon}\left(t^{\prime}\right)=\varepsilon^{2+(1-\alpha)(2-\beta)} \int_{0}^{t^{\prime} / \varepsilon^{2}} \mathrm{~d} \theta \int_{0}^{\theta} \mathrm{d} \sigma \sin \left(\varepsilon^{b} \sigma\right) r(\sigma)
$$

To conclude the proof, we shall prove that

$$
M^{\varepsilon}\left(t^{\prime}\right) \stackrel{\varepsilon \rightarrow 0}{\longrightarrow} c_{0} t^{\prime} \int_{0}^{\infty} \sigma^{-\alpha} \sin (\sigma) \mathrm{d} \sigma .
$$

By an integration by part we get

$$
M^{\varepsilon}\left(t^{\prime}\right)=\varepsilon^{(1-\alpha)(2-\beta)} t^{\prime} \int_{0}^{t^{\prime} / \varepsilon^{2}} \mathrm{~d} \theta \sin \left(\varepsilon^{b} \theta\right) r(\theta)-\varepsilon^{2+(1-\alpha)(2-\beta)} \int_{0}^{t^{\prime} / \varepsilon^{2}} \mathrm{~d} \theta \sin \left(\varepsilon^{b} \theta\right) \theta r(\theta) .
$$

The two terms in the right-hand side can be treated as $I_{1,1}^{\varepsilon}(s, t)$ and $I_{1,2}^{\varepsilon}(s, t)$, respectively, in the proof of Lemma 4. This concludes the proof.

\subsection{A remark on rough paths theory}

We conclude this section by a remark on rough paths theory. In the 70's, Doss and Sussmann worked on the gap between ordinary differential equations and stochastic differential equations [4,30]. One their most important results deals with the continuity of the Itô's map. We consider $x$ to be the solution of the ordinary differential equation driven by the continuous noise $w$ :

$$
\mathrm{d} x(t)=G(x(t)) \mathrm{d} t+F(x(t)) \mathrm{d} w(t), \quad x(t=0)=x_{0} .
$$

Their result, roughly, is the following: if $w$ is a one-dimensional noise, then the Itô's map $\mathcal{I}: w \mapsto x$ is continuous with respect to the uniform norm, but if $w$ is a multi-dimensional noise, then $\mathcal{I}$ is not continuous with respect to the uniform norm in the general case. So, one of the main interests of the rough paths theory is to establish the continuity of $\mathcal{I}$ with a topology stronger than the uniform topology. The conclusion of this remark is the following: we could prove Theorems 2 and 3 with the theory of Doss and Sussmann, but the rough paths theory of Lyons makes it possible to generalize these results to multi-dimensional cases as in Theorem 5 .

\section{Applications}

In this section we give applications in two physical situations: the classical random harmonic oscillator and the quantum random harmonic oscillator. Both systems have been extensively studied because they are great pedagogical tools and they can be encountered in many physical frameworks $[2,8,21,24,28]$.

\subsection{The classical random harmonic oscillator}

We consider the classical harmonic oscillator in a random field $\eta^{\kappa} m\left(t / \eta^{\tau}\right)$ where $m$ is a stationary, continuous, Gaussian, and centered random process which satisfies $(\mathcal{H} 3)$ or $(\mathcal{H} 4), \eta$ a small dimensionless parameter, $\tau>0$ and $\kappa=(3+\tau(1 / 2-\alpha)) / 2$. The evolution is described by the ordinary differential equation:

$$
\left\{\begin{array}{l}
\frac{d^{2} y}{\mathrm{~d} t^{2}}(t)+\omega^{2} y(t)=\eta^{\kappa} m\left(\frac{t}{\eta^{\tau}}\right) \text { for } t>0 \\
y(t=0)=y_{0} \\
\frac{\mathrm{d} y}{\mathrm{~d} t}(t=0)=y_{0}^{\prime} .
\end{array}\right.
$$


The aim of this section is to compute and to study the asymptotic behavior of the energy $H$ of the random harmonic oscillator:

$$
H(t)=|y(t)|^{2}+\frac{1}{\omega^{2}}\left|\frac{\mathrm{d} y}{\mathrm{~d} t}(t)\right|^{2}
$$

We let

$$
u(t):=y(t)+\frac{1}{i \omega} \frac{\mathrm{d} y}{\mathrm{~d} t}(t)
$$

so

$$
\left\{\begin{array}{l}
\frac{\mathrm{d} u}{\mathrm{~d} t}(t)=i \omega u(t)-\frac{i \eta^{\kappa}}{\omega} m\left(\frac{t}{\eta^{\tau}}\right) \text { for } t>0 \\
u(t=0)=y_{0}+\frac{1}{i \omega} y_{0}^{\prime}
\end{array}\right.
$$

We perform a change of variable

$$
x(t):=\mathrm{e}^{-i \omega t} u(t)
$$

so that, instead of (25), we can consider

$$
\left\{\begin{array}{l}
\frac{\mathrm{d} x}{\mathrm{~d} t}(t)=-\frac{i \eta^{\kappa}}{\omega} \mathrm{e}^{-i \omega t} m\left(\frac{t}{\eta^{\tau}}\right) \text { for } t>0 \\
x(t=0)=y_{0}+\frac{1}{i \omega} y_{0}^{\prime}
\end{array}\right.
$$

Then

$$
x(t)=y_{0}+\frac{1}{i \omega} y_{0}^{\prime}+\frac{\eta^{\kappa}}{\omega} \int_{0}^{t}\left[\sin \left(t^{\prime} \omega\right)+i \cos \left(t^{\prime} \omega\right)\right] m\left(\frac{t^{\prime}}{\eta^{\tau}}\right) \mathrm{d} t^{\prime}
$$

and

$$
\begin{aligned}
H(t)= & y_{0}^{2}+2 y_{0} \frac{\eta^{\kappa}}{\omega} \int_{0}^{t} \sin \left(t^{\prime} \omega\right) m\left(\frac{t^{\prime}}{\eta^{\tau}}\right) \mathrm{d} t^{\prime}+\frac{\eta^{2 \kappa}}{\omega^{2}}\left(\int_{0}^{t} \sin \left(t^{\prime} \omega\right) m\left(\frac{t^{\prime}}{\eta^{\tau}}\right) \mathrm{d} t^{\prime}\right)^{2} \\
& +\frac{y_{0}^{\prime 2}}{\omega^{2}}-2 y_{0}^{\prime} \frac{\eta^{\kappa}}{\omega^{2}} \int_{0}^{t} \cos \left(t^{\prime} \omega\right) m\left(\frac{t^{\prime}}{\eta^{\tau}}\right) \mathrm{d} t^{\prime}+\frac{\eta^{2 \kappa}}{\omega^{2}}\left(\int_{0}^{t} \cos \left(t^{\prime} \omega\right) m\left(\frac{t^{\prime}}{\eta^{\tau}}\right) \mathrm{d} t^{\prime}\right)^{2}
\end{aligned}
$$

We get the main result of this section by letting $\varepsilon=\eta^{1+\tau / 2}$ and applying Theorem 6 .

Theorem 7. Let us denote the rescaled energy $H^{\eta}(t)=H\left(t / \eta^{2}\right)$. Then, when $\eta$ goes to $0, H^{\eta}$ converges to $H^{0}$ in distribution in $\mathcal{C}([0,1], \mathbb{R})$ where $H^{0}$ reads as

$$
H^{0}(t)=y_{0}^{2}+\frac{y_{0}^{\prime 2}}{\omega^{2}}+2|\omega|^{\alpha} C_{0}\left(y_{0} W_{1}(t)+\frac{y_{0}^{\prime}}{\omega} W_{2}(t)\right)+|\omega|^{2 \alpha} C_{0}^{2}\left(W_{1}(t)^{2}+W_{2}(t)^{2}\right)
$$

where $W_{1}$ and $W_{2}$ are two independent Brownian motions, $C_{0}^{2}=c_{0} \cos ((1-\alpha) \pi / 2) \Gamma(2-\alpha) /(1-\alpha)$.

One thus obtains linear diffusive growth of the energy, which is standard in the case of strongly decaying covariance function [2]. However the dependence of the energy growth with respect to the oscillation frequency $\omega$ exhibits a non-standard structure $\left(\mathbb{E}\left[H^{0}(t)\right]=H(0)+2|\omega|^{2 \alpha} C_{0}^{2} t\right)$. 


\subsection{The quantum random harmonic oscillator}

\subsubsection{The nonperturbed model}

The state vector $\psi(t, x)$ of a particle of mass $M$ in a quadratic trapping potential obeys the Schrödinger equation:

$$
i \hbar \frac{\partial \psi}{\partial t}(t, x)=-\frac{\hbar^{2}}{2 M} \frac{\partial^{2} \psi}{\partial x^{2}}(t, x)+\frac{1}{2} M \omega^{2} x^{2} \psi(t, x),
$$

where $\omega$ is the oscillation frequency and $\hbar$ is the Planck constant. We multiply the spatial coordinate $x$ by $\sqrt{M \omega / \hbar}$ and the time by $\omega$ to rewrite $(27)$ in the dimensionless form:

$$
2 i \frac{\partial \psi}{\partial t}(t, x)=-\frac{\partial^{2} \psi}{\partial x^{2}}(t, x)+x^{2} \psi(t, x) .
$$

\subsubsection{The particle in a random force field}

We assume the particle to be in an external random force field which derives from the potential $\eta^{\kappa} x m\left(t / \eta^{\tau}\right)$. $m$ is a stationary, continuous, Gaussian, and centered process which satisfies $(\mathcal{H} 3)$ or $(\mathcal{H} 4), \eta$ is a small dimensionless parameter, $\tau>0$ and $\kappa=(3+\tau(1 / 2-\alpha)) / 2$. The state vector $\psi$ obeys the partial differential equation:

$$
\left\{\begin{array}{l}
2 i \frac{\partial \psi}{\partial t}(t, x)=-\frac{\partial^{2} \psi}{\partial x^{2}}(t, x)+x^{2} \psi(t, x)+\eta^{\kappa} x m\left(\frac{t}{\eta^{\tau}}\right) \psi(t, x) \\
\psi(t=0, x)=\psi_{0}(x)
\end{array}\right.
$$

This can model the motion of a charged particle in a random external electric field. Our aim is to study the asymptotic behavior of the energy in a long time scale. We define the energy

$$
H(t)=\int_{-\infty}^{+\infty}\left(\left|\frac{\partial \psi}{\partial x}(t, x)\right|^{2}+x^{2}|\psi(t, x)|^{2}\right) \mathrm{d} x
$$

The main theorem of this section is the following.

Theorem 8. Let us denote the rescaled energy $H^{\eta}(t)=H\left(t / \eta^{2}\right)$. If $H(0)<+\infty$, then, when $\eta$ goes to 0 , $H^{\eta}$ converge to $H^{0}$ in distribution in $\mathcal{C}([0,1], \mathbb{R})$ where $H^{0}$ reads

$$
H^{0}(t)=H(0)+C_{0}\left(R W_{1}(t)+I W_{2}(t)\right)+E_{0}^{2} C_{0}^{2}\left(W_{1}(t)^{2}+W_{2}(t)^{2}\right)
$$

where $W_{1}$ and $W_{2}$ are two independent Brownian motions, $C_{0}^{2}=c_{0} \cos ((1-\alpha) \pi / 2) \Gamma(2-\alpha) /(1-\alpha)$, $R=$ $\int_{-\infty}^{+\infty} x\left|\psi_{0}(x)\right|^{2} \mathrm{~d} x, I$ is the imaginary part of $\int_{-\infty}^{+\infty}\left(\partial \psi_{0}(x) / \partial x\right) \overline{\psi_{0}(x)} \mathrm{d} x$, and $E_{0}^{2}=\int_{-\infty}^{+\infty}\left|\psi_{0}(x)\right|^{2} \mathrm{~d} x$.

Proof. We rewrite the energy of the perturbed quantum harmonic oscillator with perturbation of the form $\eta^{\kappa} x m\left(t / \eta^{\tau}\right) \psi(t, x)$ in term of the energy of an equivalent classical harmonic oscillator with additive noise [2]. As a result the energy reads:

$$
\begin{aligned}
H^{\eta}(t)= & H(0)+R \eta^{\kappa} \int_{0}^{t / \eta^{2}} \sin \left(t^{\prime}\right) m\left(\frac{t^{\prime}}{\eta^{\tau}}\right) \mathrm{d} t^{\prime}+E_{0}^{2} \eta^{2 \kappa}\left(\int_{0}^{t / \eta^{2}} \sin \left(t^{\prime}\right) m\left(\frac{t^{\prime}}{\eta^{\tau}}\right) \mathrm{d} t^{\prime}\right)^{2} \\
& +I \eta^{\kappa} \int_{0}^{t / \eta^{2}} \cos \left(t^{\prime}\right) m\left(\frac{t^{\prime}}{\eta^{\tau}}\right) \mathrm{d} t^{\prime}+E_{0}^{2} \eta^{2 \kappa}\left(\int_{0}^{t / \eta^{2}} \cos \left(t^{\prime}\right) m\left(\frac{t^{\prime}}{\eta^{\tau}}\right) \mathrm{d} t^{\prime}\right)^{2} .
\end{aligned}
$$

We let $\varepsilon=\eta^{1+\tau / 2}$ and we apply Theorem 6 to complete the proof. 


\section{Appendix: technical lemmas}

The proofs of the main results are based on the tightness in the space of geometric rough paths. Moreover we need to compute the $p$-variation for $p>1$. So, we remember the reader with the two following important lemmas. The first one can be found for instance in [15], the second one in [13,15]. Finally we establish a tightness result for Gaussian geometric rough paths.

Lemma 11. Let $q>1$ and $\left(\widetilde{x}^{\varepsilon}\right)_{\varepsilon>0}=\left(\widetilde{x}_{1}^{\varepsilon}, \ldots, \widetilde{x}_{[q]}^{\varepsilon}\right)_{\varepsilon>0}$ a family of random geometric rough paths of finite $q$-variation such that $\left(\left(\widetilde{x}^{\varepsilon}(0, t)\right)_{t \in[0,1]}\right)_{\varepsilon>0}$ is tight in the space of continuous functions on $[0,1]$ and for all $j=1, \ldots,[q]$

$$
\lim _{A \rightarrow+\infty} \sup _{\varepsilon>0} \mathbb{P}\left[\left(\sup _{D} \sum_{l}\left|\widetilde{x}_{j}^{\varepsilon}\left(t_{l-1}, t_{l}\right)\right|^{q / j}\right)^{j / q}>A\right]=0
$$

where $\sup _{D}$ runs over on all finite subdivisions $\left\{t_{l}\right\}_{l}$ of $[0,1]$. Then $\left(\widetilde{x}^{\varepsilon}\right)_{\varepsilon>0}$ is tight in the space of geometric rough paths for the distance $d_{p}$ for all $p>q$.

Lemma 12. For all $n \in \mathbb{N}$ and all $k=0,1, \ldots, 2^{n}$, we let $t_{k}^{n}:=k / 2^{n}$. Let $q>1$ and $\widetilde{x}$ be a geometric rough path of finite $q$-variation. Then there exist two positive constants $\gamma, \lambda$ which do not depend on $\widetilde{x}$ such that

$$
\sup _{D} \sum_{l}\left|\widetilde{x}\left(t_{l-1}, t_{l}\right)\right|^{q} \leq \lambda \sum_{n=1}^{+\infty} n^{\gamma} \sum_{k=1}^{2^{n}}\left|\widetilde{x}\left(t_{k-1}^{n}, t_{k}^{n}\right)\right|^{q} .
$$

Lemma 13 (tightness result for Gaussian geometric rough paths). Let $p>1$ and $\left(\widetilde{x}^{\varepsilon}\right)_{\varepsilon>0}=\left(\widetilde{x}_{1}^{\varepsilon}, \ldots, \widetilde{x}_{[p]}^{\varepsilon}\right)_{\varepsilon>0}$ a family of Gaussian random geometric rough paths (that is to say $\widetilde{x}_{1}^{\varepsilon}$ is Gaussian) in $\Omega_{p}(\mathbb{R})$. We assume that there exists two constants $C>0$ and $\eta>2 / p$ such that

$$
\mathbb{E}\left[\widetilde{x}_{1}^{\varepsilon}(s, t)^{2}\right] \leq C|t-s|^{\eta}
$$

for all $s$ and $t$ in $[0,1]$. Then, $\left(\widetilde{x}^{\varepsilon}\right)_{\varepsilon>0}$ is tight for the distance $d_{p}$.

Proof. Let $q \in] 2 / \eta, p[$. Note that for a Gaussian variable, we can compare high-order moments with secondorder moments, so it is possible to work with second-order moments here. Using Kolmogorov criteria [1], the fact that $\widetilde{x}_{1}^{\varepsilon}$ is Gaussian and $(31)$ we get that $\left(\left(\widetilde{x}^{\varepsilon}(0, t)\right)_{t \in[0,1]}\right)_{\varepsilon>0}$ is tight in the space of continuous functions on $[0,1]$. Hence, following Lemma 11, it rest to show $(30)$ for $\left(\widetilde{x}^{\varepsilon}\right)_{\varepsilon>0}$. By Markov inequality and Lemma 12 :

$$
\mathbb{P}\left[\left(\sup _{D} \sum_{l}\left|\widetilde{x}_{j}^{\varepsilon}\left(t_{l-1}, t_{l}\right)\right|^{q / j}\right)^{j / q}>A\right] \leq \frac{1}{A^{q / j}} \sum_{n=1}^{+\infty} n^{\gamma}\left(2^{n}\right)^{1-q \eta / 2}
$$

for all $j=1, \ldots,[q]$. Taking the sup on $\varepsilon$ and letting $A \rightarrow \infty$ completes the proof.

Acknowledgements. I would like to thank Professors Laure Coutin and Josselin Garnier for their helpful suggestions and comments on this work. I would also like to thank Professor Antoine Lejay for interesting discussions on the rough paths theory.

\section{REFERENCES}

[1] P. Billingsley, Convergence of Probability Measures. Wiley (1968).

[2] A. Bunimovich, H.R. Jauslin, J.L. Lebowitz, A. Pellegrinotti and P. Nielaba, Diffusive energy growth in classical and quantum driven oscillators. J. Stat. Phys. 62 (1991) 793-817.

[3] L. Coutin and Z. Qian, Stochastic analysis, rough path analysis and fractional Brownian motions. Prob. Th. Related Fields 122 (2002) 108-140. 
[4] H. Doss, Liens entre équations différentielles stochastiques et ordinaires. Ann. Inst. H. Poincaré 13 (1977) 99-125.

[5] S.N. Ethier and T.G. Kurtz, Markov processes, characterization and convergence. Wiley, New York (1986).

[6] J.P. Fouque, G. Papanicolaou and R. Sircar, Derivatives in Financial Markets with Stochastic Volatility. Cambridge University Press (2000).

[7] J. Garnier, A multi-scaled diffusion-approximation theorem. Applications to wave propagation in random media. ESAIM: PS 1 (1997) 183-206.

[8] J. Garnier, Asymptotic behavior of the quantum harmonic oscillator driven by a random time-dependent electric field. J. Stat. Phys. 93 (1998) 211-241.

[9] J. Garnier, Scattering, spreading, and localization of an acoustic pulse by a random medium, in Three Courses on Partial Differential Equations, E. Sonnendrücker Ed. Walter de Gruyter, Berlin (2003) 71-123.

[10] J. Garnier and R. Marty, Effective pulse dynamics in optical fibers with polarization mode dispersion. Preprint, submitted to Wave Motion.

[11] R.Z. Khasminskii, A limit theorem for solutions of differential equations with random right hand side. Theory Probab. Appl. 11 (1966) 390-406.

[12] H.J. Kushner, Approximation and weak convergence methods for random processes. MIT Press, Cambridge (1994).

[13] M. Ledoux, T. Lyons and Z. Qian, Lévy area of Wiener processes in Banach spaces. Ann. Probab. 30 (2002) 546-578.

[14] M. Ledoux, Z. Qian and T. Zhang, Large deviations and support theorem for diffusion processes via rough paths. Stoch. Proc. Appl. 102 (2002) 265-283.

[15] A. Lejay, An introduction to rough paths, in Séminaire de Probabilités XXXVII. Lect. Notes Math. Springer-Verlag (2003).

[16] T. Lyons, Differential equations driven by rough signals. Rev. Mat. Iberoamer. 14 (1998) 215-310.

[17] T. Lyons, Differential equations driven by rough signals (I): an extension of an inequality of L.C. Young. Math. Res. Lett. 1 (1994) 451-464.

[18] T. Lyons and Z. Qian, Flow equations on spaces of rough paths. J. Funct. Anal. 149 (1997) 135-159.

[19] T. Lyons and Z. Qian, System control and rough paths. Oxford Mathematical Monographs. Oxford University Press (2002).

[20] R. Marty, Théorème limite pour une équation différentielle à coefficient aléatoire à mémoire longue. C. R. Acad. Sci. Paris, Ser. I 338 (2004).

[21] A. Messiah, Quantum Mechanics. North Holland, Amsterdam (1962).

[22] D. Middleton, Introduction to statistical communication theory. Mc Graw Hill Book Co., New York (1960).

[23] G. Papanicolaou, Waves in one dimensional random media, in École d'été de Probabilités de Saint-Flour, P.L. Hennequin Ed. Springer. Lect. Notes Math. (1988) 205-275.

[24] G. Papanicolaou and J.B. Keller, Stochastic differential equations with two applications to random harmonic oscillators and waves in random media. SIAM J. Appl. Math. 21 (1971) 287-305.

[25] G. Papanicolaou and W. Kohler, Asymptotic theory of mixing stochastic ordinary differential equations. Comm. Pure Appl. Math. 27 (1974) 641-668.

[26] G. Papanicolaou, D.W. Stroock and S.R.S.Varadhan, Martingale approach to some limit theorem, in Statistical Mechanics and Dynamical systems, D. Ruelle Ed. Duke Turbulence Conf., Duke Univ. Math. Series III, Part IV (1976) 1-120.

[27] G. Samorodnitsky and M.S. Taqqu, Stable non-Gaussian random processes. Chapman and Hall (1994).

[28] L.I. Schiff, Quantum Mechanics. Mac Graw Hill, New York (1968).

[29] K. Sølna, Acoustic Pulse Spreading in a Random Fractal. SIAM J. Appl. Math. 63 (2003) 1764-1788.

[30] H.J. Sussmann, On the gap between deterministic and stochastic ordinary differential equations. Ann. Prob. 6 (1978) $19-41$. 